\title{
Propiedades PSICOMÉTRICAS DE LA VERSIÓN CORTA DEL INVENTARIO DE INTELIGENCIA EMOCIONAL (EQi-SF) EN POBLACIÓN MEXICANA
}

\author{
Norma A. Ruvalcaba-Romero \\ https://orcid.org/0000-0001-9209-8751 \\ Mercedes Gabriela Orozco-Solís \\ https://orcid.org/0000-0002-0648-8233 \\ HÉCtOR RuBÉn BRAVO-ANDRADE \\ https://orcid.org/0000-0002-0498-8410 \\ Centro Universitario de Ciencias de la Salud, Universidad de Guadalajara, México \\ Correo electrónico: norma.ruvalcaba@academicos.udg.mx
}

Recibido: 19 de octubre del 2020 / Aceptado: 26 de octubre del 2020

doi: https://doi.org/10.26439/persona2020.n023(2).4885

\begin{abstract}
Resumen. La inteligencia emocional es considerada como un factor protector contra una variedad de problemáticas psicosociales. Debido a la relevancia de este constructo, se ha diseñado una variedad de instrumentos para su evaluación, entre los que destaca el Inventario de Inteligencia Emocional (EQi-SF). El objetivo de este trabajo es identificar las propiedades psicométricas del EQi-SF en población mexicana. El estudio se llevó a cabo en una muestra de 829 bachilleres en el estado de Jalisco, México. Se aplicó la versión de 28 ítems del EQi-SF con una estructura Likert de 5 puntos que evalúa cuatro factores: competencia intrapersonal, competencia interpersonal, manejo del estrés y adaptabilidad. Se llevaron a cabo análisis factoriales, a partir de los cuales se corrobora una estructura factorial de cuatro dimensiones con índices de ajuste adecuados, así como niveles de confiabilidad de aceptables a buenos. Se contrastan los resultados a la luz de estudios previos y se discuten implicaciones y recomendaciones.
\end{abstract}

Palabras clave: inteligencia emocional / instrumentos / validación / población mexicana 


\title{
PSYCHOMETRIC PROPERTIES OF THE EMOTIONAL QUOTIENT INVENTORY-SHORT FORM (EQI-SF) IN MEXICANS
}

\begin{abstract}
Emotional intelligence is considered a protective factor against a variety of psychosocial problems. Due to the significance of this construct, an array of instruments have been designed for its evaluation, among which the Emotional Quotient Inventory-Short Form (EQi-SF) stands out. This work aims to identify the psychometric properties of the EQi-SF in Mexicans. The study was carried out in a sample of 829 high school graduates from the state of Jalisco, Mexico. The 28-item version of the EQi-SF was administered with a 5-point Likert scale that assessed 4 factors: intrapersonal competence, interpersonal competence, stress management and adaptability. Factorial analyses were performed, thus confirming a four-dimension factorial structure with adequate fit indices and acceptable-to-good reliability levels. The results are compared with previous studies, and the implications and recommendations are discussed.
\end{abstract}

Keywords: emotional intelligence / instruments / validation / Mexican population 


\section{INTRODUCCIÓN}

La inteligencia emocional es un constructo que ha tenido relevancia desde las primeras investigaciones realizadas al respecto, debido principalmente a la relación que mantiene con diversos recursos psicológicos que influyen en el ajuste psicosocial. En la actualidad, es considerada un factor protector frente a problemáticas como la depresión, ansiedad, suicidio, bajo desempeño académico y laboral, estrés, entre otras (Barraza-López, Muñoz-Navarro y Behrens-Pérez, 2017; Gómez-Romero, Limonero, Toro, Montes-Hidalgo y Tomás-Sábado, 2018; Pincay-Aguilar, Candelario-Suarez y Castro-Guevara, 2018; Quiliano y Quiliano, 2020). Asimismo, se reporta como un factor predictor de la autoestima, resiliencia, habilidad de solución de problemas, conducta prosocial, bienestar, satisfacción con la vida, entre otras variables (Cejudo, López-Delgado y Rubio, 2016; Ros, Filella, Ribes y Pérez-Escoda, 2017; Ruvalcaba, Gallegos y Fuerte, 2017; Samper, Mestre y Malonda, 2014; Serrano y Andreu, 2016).

Debido a la relevancia de este constructo, actualmente existe una amplia variedad de instrumentos de medición diseñados para su evaluación, los cuales se basan principalmente en dos modelos. La primera propuesta parte del modelo de habilidad, el cual concibe a la inteligencia emocional como la capacidad de monitorear y distinguir las emociones, propias y de los demás, y utilizar esa información para guiar los pensamientos y conductas en la toma de decisiones (Salovey y Mayer, 1990). Los instrumentos que se basan en este enfoque incluyen medidas de habilidad, siendo evaluadas principalmente por medio de percepción de capacidades, tareas de ejecución e informes de observadores externos (Fernández-Berrocal, Extremera y Ramos, 2004; Jordan y Lawrence, 2009; Lyusin y Ovsyannikova, 2016).

En el segundo modelo, denominado mixto, se propone que la inteligencia emocional no solo comprende habilidades cognitivas, sino que incorpora una serie de rasgos de personalidad, competencias socioemocionales, aptitudes y aspectos motivacionales (Bar-On, 2006; Bar-On y Parker, 2000; Extremera, Fernández-Berrocal, Mestre y Guil, 2004). En este modelo, la inteligencia emocional implica las habilidades relacionadas con la capacidad de gestionar con eficacia los cambios del entorno, manteniendo una postura realista y flexible que permita afrontar problemas y tomar decisiones con una actitud positiva (Bar-On, 2006; Petrides et al., 2016). Los instrumentos diseñados de acuerdo con este modelo plantean medidas de autoinforme más influenciadas por rasgos de personalidad (Bar-On, 1997a; Parker, Keefer y Wood, 2011; Pérez-Fuentes, Gázquez, Mercader y Molero, 2014). En este estudio nos enfocaremos en una de las principales herramientas de medición realizadas con base en este último modelo. 


\section{EL MODELO DE BAR-ON}

De acuerdo con Bar-On (2006), la inteligencia socioemocional representa un conjunto interrelacionado de competencias socioemocionales, habilidades y facilitadores que influyen en la capacidad para comprender las emociones, expresarlas, entender lo que siente el otro, relacionarse exitosamente con los demás y afrontar las demandas del contexto. Desde esta postura, los elementos que integran la inteligencia emocional pueden agruparse en cinco grandes componentes: competencia intrapersonal, competencia interpersonal, manejo del estrés, adaptabilidad y estado de ánimo (tabla 1). Con la finalidad de evaluar la pertinencia del modelo conceptual, el autor desarrolló el Emotional Quotient Inventory (EQ-i) (Bar-On, 1997b).

Tabla 1

Componentes integrados en el modelo de Bar-On de inteligencia emocional

\begin{tabular}{|c|c|c|}
\hline Componente & Subescalas & Definición \\
\hline $\begin{array}{l}\text { Competencia } \\
\text { intrapersonal }\end{array}$ & $\begin{array}{l}\text { Autoconcepto } \\
\text { Autoconciencia emocional } \\
\text { Asertividad } \\
\text { Independencia } \\
\text { Autoactualización }\end{array}$ & $\begin{array}{l}\text { Habilidades relacionadas con el reconocimiento } \\
\text { de las propias emociones, la comprensión de } \\
\text { fortalezas y debilidades personales, la expresión de } \\
\text { sentimientos, pensamientos y necesidades desde } \\
\text { una postura constructiva }\end{array}$ \\
\hline $\begin{array}{l}\text { Competencia } \\
\text { interpersonal }\end{array}$ & $\begin{array}{l}\text { Empatía } \\
\text { Responsabilidad social } \\
\text { Relaciones interpersonales }\end{array}$ & $\begin{array}{l}\text { Competencias involucradas en el reconocimiento } \\
\text { de las emociones y necesidades de los demás, } \\
\text { y en la generación de relaciones cooperativas, } \\
\text { constructivas y mutuamente satisfactorias }\end{array}$ \\
\hline Manejo del estrés & $\begin{array}{l}\text { Tolerancia al estrés } \\
\text { Control de impulsos }\end{array}$ & $\begin{array}{l}\text { Capacidades relacionadas con la tolerancia y el } \\
\text { control de impulsos, hacen referencia al manejo } \\
\text { efectivo y constructivo de las emociones }\end{array}$ \\
\hline Adaptabilidad & $\begin{array}{l}\text { Estrategia de solución de } \\
\text { problemas } \\
\text { Prueba de realidad } \\
\text { Flexibilidad }\end{array}$ & $\begin{array}{l}\text { Habilidades que permiten el afrontamiento de los } \\
\text { problemas desde una postura realista, flexible y } \\
\text { enfocada a la solución de las dificultades personales } \\
\text { e interpersonales }\end{array}$ \\
\hline Estado de ánimo & $\begin{array}{l}\text { Optimismo } \\
\text { Felicidad }\end{array}$ & $\begin{array}{l}\text { Competencias relacionadas con la capacidad } \\
\text { de mantener una actitud optimista frente a los } \\
\text { problemas y de generar emociones positivas }\end{array}$ \\
\hline
\end{tabular}

Fuente: adaptado de Bar-On (2006)

Elaboración propia 


\section{Inventario de Inteligencia Emocional (EQ-i)}

En su versión original, el EQ-i mantiene un formato de autoinforme y consta de 133 ítems divididos en cinco dimensiones y 15 subescalas (tabla 1). Las opciones de respuesta se presentan en escala tipo Likert de 5 puntos $(1=$ de acuerdo a $5=$ en desacuerdo). La forma de calificación permite una puntuación total y una específica para las cinco dimensiones y sus subescalas. Las puntuaciones altas indican un mayor desarrollo de la competencia evaluada y un mejor funcionamiento en el afrontamiento de los problemas. $\mathrm{El}$ instrumento también analiza cuatro indicadores que miden las respuestas relacionadas con la deseabilidad social (Bar-On, 1997b).

La primera evaluación de las propiedades psicométricas del instrumento fue realizada con una muestra de Canadá y Estados Unidos, en la que se probó la validez de constructo que respaldó la estructura de cinco dimensiones y 15 subescalas (Bar-On, 1997b). Los análisis confirmatorios validaron la estructura factorial propuesta previamente, y encontraron también buen ajuste en una construcción de cinco dimensiones y 10 subescalas, que excluye los factores: independencia, autoactualización, responsabilidad social, optimismo y felicidad (Bar-On, 1997a). Con base en esos resultados, se concluyó que las 10 subescalas que mantuvieron validez representan competencias clave de la inteligencia emocional, mientras que los cinco elementos restantes podrían ser considerados como actitudes facilitadoras (Bar-On, 1997a).

Los resultados de distintas evaluaciones de las propiedades psicométricas del inventario respaldan la validez y confiabilidad del modelo de cinco dimensiones, siendo adaptado y validado para más de treinta poblaciones donde solo se reportan algunas diferencias en la distribución de las subescalas (Bar-On, 2006; Dawda y Hart, 2000; Rivera, Pons, Rosario-Hernández y Ortiz, 2008; Stanimirovic y Hanrahan, 2012; Weerdt y Rossi, 2012; Ugarriza, 2001). De manera general, la validez convergente y divergente ha sido comprobada con variables como alexitimia, razonamiento verbal, características de personalidad del NEO-FFI, depresión, intensidad de la experiencia afectiva, somatización, entre otras (Dawda y Hart, 2000; Parker, Taylor y Bagby, 2001; Regner, 2008).

\section{Inventario de Inteligencia Emocional-Forma Corta (EQ-i:S)}

A partir de la evidencia recabada sobre las propiedades psicométricas del EQ-i y de la relevancia encontrada con relación a otras variables, se rescató la necesidad de estructurar versiones más cortas y fáciles de aplicar del inventario. Por esa razón, Bar-On (2002) propuso una versión corta (EQ-i:S) que cuenta con 35 ítems divididos en cuatro dimensiones: intrapersonal (10 ítems), interpersonal (10 ítems), manejo del estrés (8 ítems)

y adaptabilidad (7 ítems). En esta versión se propone que la subescala estado de ánimo (10 ítems) representa un factor de segundo orden, por lo cual no siempre se incluye en 
los análisis de propiedades psicométricas. Asimismo, se conserva una subescala que aborda la impresión positiva (6 ítems) relativa a la deseabilidad social. El formato de respuesta se presenta en escala tipo Likert de 5 puntos ( $1=$ nunca a $5=$ siempre). De igual manera, permite obtener una puntuación total de inteligencia emocional y puntuaciones específicas para cada dimensión.

De acuerdo con Bar-On (2002), en la primera evaluación de las propiedades psicométricas de la versión corta se corroboró la estructura de cuatro dimensiones primarias y un factor de segundo orden. Las primeras evaluaciones de la versión corta reportaron propiedades aceptables, encontrando correlaciones de medias a altas con las dimensiones de la versión larga (.73 a .97 para ambos sexos) y niveles de consistencia interna aceptables en las cinco dimensiones (Bar-On, 2002).

En uno de los análisis confirmatorios realizados para la versión corta, se exploraron las propiedades del modelo con cuatro dimensiones y un factor de segundo orden, correspondiente al estado de ánimo, y se obtuvieron índices de ajuste adecuados (Root Mean Square Error of Approximation, RMSEA = .57; Standardised Root Mean Residual, SRMR = .57; Comparative Fit Index, CFI = .922). Asimismo, se comprobó el ajuste de una estructura con solo las cuatro dimensiones principales (RMSEA $=.56, \mathrm{SRMR}=.56, \mathrm{CFI}=.924$ ). A pesar de que ambos modelos presentaron niveles de ajuste aceptables, los resultados indicaron que el segundo modelo mantuvo mejor parsimonia y conservó la misma estructura en la distribución de los ítems (Parker et al., 2011).

El EQ-i:S también ha sido adaptado y validado para distintas poblaciones. En el estudio realizado por Görgens-Ekermans, Saklofske, Austin y Stough (2011), se analizó la validez del modelo de cuatro dimensiones y un factor de segundo orden en muestras de Australia, Sudáfrica, Canadá y Escocia. Los mejores niveles de ajuste se encontraron en la muestra canadiense (RMSEA $=.053, \mathrm{SRMR}=.052, \mathrm{CFI}=.97$ ) y la australiana (RMSEA $=.056$, SRMR $=.053, \mathrm{CFI}=.98)$. Las muestras de Sudáfrica (RMSEA $=.067$, $\mathrm{SRMR}=.057, \mathrm{CFI}=.97)$ y Escocia (RMSEA $=.066, \mathrm{SRMR}=.065, \mathrm{CFI}=.97)$ presentaron mayores problemas de ajuste. Por su parte, los coeficientes de consistencia interna se mantuvieron entre .784 a .923 para las cinco dimensiones evaluadas en las cuatro muestras. La distribución de los ítems fue congruente con el modelo original en los cuatro grupos.

La primera validación del EQ-i:S al español fue realizada por López-Zafra, Pulido y Berrios (2014), quienes llevaron a cabo un proceso de adaptación transcultural para estudiantes universitarios españoles con la versión de cuatro dimensiones. Los resultados finales del análisis factorial exploratorio indicaron que la estructura de cuatro factores explica el $40 \%$ de la varianza. Durante el desarrollo del análisis se eliminaron 7 ítems debido a que presentaron cargas factoriales menores a .40 y cargas factoriales secundarias menores a .30, criterio establecido por los autores. La distribución de los 
28 ítems restantes en los cuatro factores extraídos coincidió con la estructura original de las cuatro dimensiones del modelo: intrapersonal (8 ítems), interpersonal (7 ítems), manejo del estrés (8 ítems) y adaptabilidad (5 ítems).

Los autores también realizaron análisis factorial confirmatorio sobre la estructura encontrada, reportando indicadores de ajuste aceptables (RMSEA $=.04$, SRMR $=.03$, $\mathrm{CFI}=.91)$. Los cuatro factores presentaron correlaciones significativas entre sí, con valores que indican una magnitud moderada (.21 a .42). Finalmente, se llevó a cabo un análisis de validez convergente con los resultados obtenidos de la aplicación del Trait Meta Mood Scale (TMMS-24), en el que se hallaron correlaciones positivas y significativas entre la mayor parte de las dimensiones de ambos instrumentos.

En población española, esta adaptación del EQ-i:S ha sido usada para evaluar la relación de la inteligencia emocional con variables como el optimismo, pesimismo, comportamiento prosocial, satisfacción vital, resiliencia, entre otras (Gavín-Chocano, 2018; Gavín-Chocano y Molero, 2020; Martí-Vilar, Serrano-Pastor y González Sala, 2019). Esta misma versión del EQ-i:S ha sido aplicada en estudiantes chilenos para explorar sus niveles de competencias socioemocionales, demostrando adecuados coeficientes de confiabilidad (Castellanos y López, 2018). Por su parte, en México se ha utilizado para relacionar las competencias socioemocionales con indicadores como la ansiedad en poblaciones de mujeres, manteniendo adecuados índices de confiabilidad (Alva, Ruvalcaba, Orozco y Bravo, 2019).

Como es posible observar, la adaptación del EQ-i:S realizada por López-Zafra et al. (2014) mantiene propiedades psicométricas sólidas para población española y ha permitido ampliar el análisis de la relación de la inteligencia emocional con distintos recursos psicológicos importantes para el bienestar. Los resultados de los pocos estudios que han retomado esta adaptación en otros países hispanohablantes demuestran que mantiene consistencia y podría ser una herramienta adecuada para la evaluación de la inteligencia emocional. Sin embargo, en la actualidad no se cuenta con una evaluación de la validez que presenta esta versión en español al ser aplicada en poblaciones distintas a la española. Por lo tanto, el objetivo de este trabajo es identificar las propiedades psicométricas del EQi-SF en población mexicana.

\section{MÉTODO}

\section{Participantes}

La muestra estuvo constituida por 829 estudiantes de bachillerato adscritos a 39 escuelas preparatorias de la Zona Metropolitana de Guadalajara (57 \%) y preparatorias regionales del estado de Jalisco (43\%). El muestreo fue por conveniencia. Todos los 
participantes eran mayores de edad, $46 \%$ hombres y $54 \%$ mujeres, con un rango de edad entre 18 y 25 años (media $=18,45$; DT $=1,14$ ).

\section{Instrumentos}

- Datos sociodemográficos: se preguntó por el sexo y la edad de los participantes, así como por la institución de adscripción.

- Inventario de Inteligencia Emocional (EQ-i, Bar-On, 2000): los reactivos fueron incluidos de la versión publicada por Ugarriza (2001), con la estructura presentada en la validación española para la versión corta realizada por López-Zafra et al. (2014). Se trata de una escala de 28 ítems con una estructura Likert de 5 puntos que evalúa cuatro factores: competencia intrapersonal, competencia interpersonal, manejo del estrés y adaptabilidad.

\section{Procedimiento}

Previo consentimiento informado, los cuestionarios fueron aplicados de manera electrónica con el apoyo de los orientadores educativos de cada uno de los planteles participantes. Los datos fueron procesados con el paquete estadístico SPSS v. 25 y el AMOS v. 16.

\section{RESULTADOS}

En primer lugar, se realizó un análisis factorial exploratorio con los 28 ítems, con el método de componentes principales y con rotación varimax; se generaron cinco factores, en los cuales los ítems correspondientes a la competencia intrapersonal se escindieron en dos factores. A fin de dar coherencia con la validación española se forzó el análisis a cuatro factores. Los resultados se muestran en la tabla 2; se incluyen, además, los índices de consistencia interna (alfa de Cronbach) de cada uno de los factores.

Tabla 2

Análisis factorial exploratorio y coeficientes de consistencia interna

\begin{tabular}{|c|c|c|c|c|}
\hline & $\begin{array}{c}\text { Manejo } \\
\text { del estrés }\end{array}$ & Intrapersonales Adaptabilidad & Interpersonales & $\begin{array}{l}\text { Alfa sin } \\
\text { el ítem }\end{array}$ \\
\hline Soy impulsivo & .789 & & & .846 \\
\hline Exploto fácilmente & .776 & & & .849 \\
\hline $\begin{array}{l}\text { Cuesta controlar } \\
\text { impulsos }\end{array}$ & .752 & & & .847 \\
\hline $\begin{array}{l}\text { Impulsividad } \\
\text { provoca problemas }\end{array}$ & .705 & & & .852 \\
\hline
\end{tabular}


(continuación)

\begin{tabular}{|c|c|c|c|c|c|}
\hline Tengo mal carácter & .694 & & & & .856 \\
\hline $\begin{array}{l}\text { Problema controlar } \\
\text { enojo }\end{array}$ & .692 & & & & .856 \\
\hline Soy impaciente & .671 & & & & .858 \\
\hline $\begin{array}{l}\text { Difícil controlar mi } \\
\text { ansiedad }\end{array}$ & .547 & & & & .866 \\
\hline $\begin{array}{l}\text { Logrado pocas } \\
\text { cosas }\end{array}$ & & .645 & & & .736 \\
\hline $\begin{array}{l}\text { Difícil tomar } \\
\text { decisiones }\end{array}$ & & .637 & & & .740 \\
\hline $\begin{array}{l}\text { Difícil describir } \\
\text { sentimientos }\end{array}$ & & .633 & & & .728 \\
\hline $\begin{array}{l}\text { Difícil entender lo } \\
\text { que siento }\end{array}$ & & .601 & & & .733 \\
\hline $\begin{array}{l}\text { Difícil expresar } \\
\text { sentimiento }\end{array}$ & & .589 & & & .751 \\
\hline $\begin{array}{l}\text { Otros decidan por } \\
\text { mí }\end{array}$ & & .561 & & & .756 \\
\hline $\begin{array}{l}\text { Incapaz expresar } \\
\text { ideas }\end{array}$ & & .521 & & & .768 \\
\hline $\begin{array}{l}\text { Difícil defender } \\
\text { derechos }\end{array}$ & & .520 & & & .755 \\
\hline $\begin{array}{l}\text { Visión general del } \\
\text { problema }\end{array}$ & & & .727 & & .710 \\
\hline $\begin{array}{l}\text { Considero } \\
\text { posibilidades }\end{array}$ & & & .689 & & .705 \\
\hline $\begin{array}{l}\text { Pienso maneras } \\
\text { posibles de solución }\end{array}$ & & & .685 & & .705 \\
\hline $\begin{array}{l}\text { Reúno información } \\
\text { ante situación difícil }\end{array}$ & & & .657 & & .725 \\
\hline $\begin{array}{l}\text { Supero dificultades } \\
\text { paso a paso }\end{array}$ & & & .507 & & .762 \\
\hline $\begin{array}{l}\text { Preocupa sucede a } \\
\text { otros }\end{array}$ & & & & .739 & .656 \\
\hline $\begin{array}{l}\text { Sensible } \\
\text { sentimientos demás }\end{array}$ & & & & .702 & .675 \\
\hline $\begin{array}{l}\text { Comprendo cómo } \\
\text { sienten otros }\end{array}$ & & & & .577 & .658 \\
\hline $\begin{array}{l}\text { Relaciones } \\
\text { significan mucho }\end{array}$ & & & & .517 & .682 \\
\hline Ayudo gente & & & & .516 & .688 \\
\hline
\end{tabular}


(continuación)

\begin{tabular}{lcccc}
\hline $\begin{array}{l}\text { Amigos cuentan } \\
\text { intimidades }\end{array}$ & & & .461 & .680 \\
Me relaciono bien & & & .438 & .694 \\
Varianza & 21.76 & 11.83 & 7.87 & 5.25 \\
\hline Alfa de Cronbach & .870 & .771 & .765 & .709 \\
\hline
\end{tabular}

Nota. Ítems resumidos

Elaboración propia

Posteriormente, se realizó el análisis factorial confirmatorio con un modelo inicial de los 28 ítems agrupados en cuatro factores, con el método de máxima verosimilitud; los índices de ajuste se muestran en la tabla 3.

Tabla 3

Índices de ajuste del análisis factorial confirmatorio

\begin{tabular}{lccccccccccc}
\hline & & \multicolumn{4}{c}{} & \multicolumn{4}{c}{ Índices de ajuste absoluto } & \multicolumn{3}{c}{ Índices de ajuste incremental } \\
\hline Modelo & $\mathrm{X}^{2}$ & $\mathrm{gl}$ & $\mathrm{x}^{2} / \mathrm{gl}$ & $\mathrm{GFI}$ & $\mathrm{AGFI}$ & $\mathrm{RMR}$ & $\mathrm{RMSEA}$ & $\mathrm{NFI}$ & $\mathrm{TLI}$ & $\mathrm{CFI}$ & $\mathrm{IFI}$ \\
1 & 1321 & 344 & 3.84 & .890 & .870 & .072 & .059 & .814 & .840 & .854 & .855 \\
2 & 997 & 338 & 2.94 & .919 & .903 & .066 & .049 & .859 & .890 & .902 & .902 \\
\hline
\end{tabular}

Elaboración propia

Como puede observarse, los índices de ajuste claramente podían ser mejorados, por lo que se procedió a incluir en un segundo modelo las covarianzas entre los siguientes pares de ítems: Sensible sentimientos demás - Preocupa sucede a otros, Soy impulsivo - Cuesta controlar impulsos, Tengo mal carácter - Exploto fácilmente, Difícil describir sentimientos - Difícil expresar sentimientos y Otros decidan por mí - Difícil tomar decisiones. Una vez incluidas las covarianzas, el modelo logró índices de bondad de ajuste adecuados.

\section{DISCUSIÓN}

El presente estudio tuvo como objetivo analizar las propiedades psicométricas del EQ-i:S en población mexicana, para lo cual se llevaron a cabo análisis factoriales exploratorios y confirmatorios.

Se encontraron evidencias de validez de constructo mediante índices de ajuste adecuados, con lo cual se corrobora la estructura de cuatro dimensiones reportada en la literatura (Görgens-Ekermans et al., 2011). Este hallazgo fortalece la evidencia 
empírica para la recomendación de interpretar los resultados del instrumento por sus dimensiones por separado y no de manera conjunta, debido a la ausencia de un factor de segundo orden que sustente un puntaje total del instrumento (Parker et al., 2011).

Asimismo, los resultados del presente estudio coinciden con los índices de la versión española y sus adaptaciones en población latinoamericana (López-Zafra et al., 2014; Ugarriza, 2001), probablemente debido a la proximidad cultural. Lo anterior permite dar cuenta de la pertinencia del instrumento al contexto mexicano. Esta versión de 28 ítems supone un modelo parsimonioso que conserva la misma estructura en la distribución (Parker et al., 2011), con la ventaja de tener un número menor de reactivos.

Al igual que en el modelo propuesto por López-Zafra et al. (2014), para mejorar el ajuste del modelo se establecieron covarianzas entre los errores de algunos ítems. Vale la pena mencionar que aquellos errores que se relacionaron correspondían a una misma dimensión, lo cual es teóricamente coincidente, al indicar dependencia entre los elementos de un mismo factor.

Por su parte, los índices de confiabilidad de las dimensiones se encontraron dentro de niveles aceptables a buenos. El menor de ellos fue competencias interpersonales $(\alpha=.70)$, y el mayor, manejo del estrés $(\alpha=.87)$. Estos resultados contrastan con lo hallado por López-Zafra et al. (2014), quienes reportan niveles aceptables que van de .70 a .78.

Una de las implicaciones de los hallazgos de este estudio es poder contar con un instrumento que permita evaluar programas encaminados a favorecer específicamente estas dimensiones y tener registros de contraste para evaluar la eficacia de dichas intervenciones mediante diseños experimentales con evaluaciones pre-post.

Como limitaciones del estudio, se reconoce que, al tratarse de población estudiantil, los resultados no pueden extenderse a otro tipo de población. También se asume que el muestreo por conveniencia limita la generalización de los resultados. Derivado de ello, se sugiere realizar investigaciones en las que se amplíe hacia una población más heterogénea, mediante muestreos aleatorizados, lo cual permitiría incluso poder elaborar baremos. Asimismo, se recomienda buscar evidencia de validez concurrente y divergente en futuros estudios.

En suma, el EQ-i:S se presenta como un instrumento multidimensional con fundamentación teórica y validez empírica confiables para la evaluación de las dimensiones: manejo del estrés, competencias intrapersonales, adaptabilidad y competencias interpersonales de la inteligencia socioemocional en población mexicana. 


\section{REFERENCIAS}

Alva, I., Ruvalcaba, N., Orozco, M., y Bravo, A. (2019). Resiliencia y competencias socioemocionales como factor preventivo de ansiedad en mujeres mexicanas. Ansiedad y Estrés, 25, 59-65. https://doi.org/10.1016/j.anyes.2019.10.003

Barraza-López, R. J., Muñoz-Navarro, N. A., y Behrens-Pérez, C. C. (2017). Relationship between emotional intelligence and depression-anxiety and stress in medical students freshmen. Revista Chilena de Neuropsiquiatría, 55(1), 18-25. https://doi. org/10.4067/S0717-92272017000100003

Bar-On, R. (1997a). The Emotional Quotient Inventory (EQ-i): a test of emotional intelligence. Toronto: Multi-Health Systems.

Bar-On, R. (1997b). The Emotional Intelligence Inventory (EQ-i). Technical manual. Toronto: Multi-Health Systems.

Bar-On, R. (2002). Bar-On Emotional Quotient Short Form (EQ-I: Short). Technical manual. Toronto: Multi-Health Systems.

Bar-On, R. (2006). The Bar-On Model of emotional-social intelligence (ESI). Psicothema, 18(Supl.), 13-25. Recuperado de http://www.psicothema.com/pdf/3271.pdf

Bar-On, R., y Parker, J. (2000). The Bar-On Emotional Quotient Inventory: Youth Version (EQ-i: YV). Technical manual. Toronto: Multi-Health Systems.

Castellanos, R., y López, I. (2018). Competencias socioemocionales en estudiantes de Tecnología Médica en una universidad privada de la Región Metropolitana de Santiago. Revista Educación en Ciencias de la Salud, 15(2), 82-86. Recuperado de http://repositorio.uchile.cl/handle/2250/145638

Cejudo, J., López-Delgado, M. L., y Rubio, M. J. (2016). Inteligencia emocional y resiliencia: su influencia en la satisfacción con la vida en estudiantes universitarios. Anuario de Psicología, 46(2), 51-57. https://doi.org/10.1016/j.anpsic.2016.07.001

Dawda, D., y Hart, S. (2000). Assessing emotional intelligence: reliability and validity of the Bar-On Emotional Quotient Inventory (EQ-i) in university students. Personality and Individual Differences, 28(4), 797-812. https://doi.org/10.1016/S01918869(99)00139-7

Görgens-Ekermans, G., Saklofske, D., Austin, E., y Stough, C. (2011). Measurement invariance and differential item functioning of the Bar-On EQ-i:S measure over Canadian, Scottish, South African and Australian samples. Personality and Individual Differences, 50, 286-290. https://doi.org/10.1016/j.paid.2010.10.004 
Extremera, N., Fernández-Berrocal, P., Mestre, J. M., y Guil, R. (2004). Medidas de evaluación de la inteligencia emocional. Revista Latinoamericana de Psicología, 36(2), 209-228. Recuperado de http://www.redalyc.org/articulo.oa?id=80536203

Fernández-Berrocal, P., Extremera, N., y Ramos, N. (2004). Validity and reliability of the Spanish modified version of the Trait Meta-Mood Scale. Psychological Reports, 94(3), 751-755. https://doi.org/10.2466/pr0.94.3.751-755

Gavín-Chocano, 0. (2018). Inteligencia emocional rasgo y su influencia sobre el optimismo disposicional en profesionales de centros de atención a personas con discapacidad intelectual. MLS Educational Research, 2(2). https://doi.org/10.29314/mlser. v2i2.81

Gavín-Chocano, O., y Molero, D. (2020). Relación entre inteligencia emocional y optimismo vs. pesimismo en trabajadores de centros para personas con discapacidad intelectual. Revista Española de Discapacidad, 8(1), 129-144. https://doi. org/10.5569/2340-5104.08.01.07

Gómez-Romero, M. J., Limonero, J. T., Toro, J., Montes-Hidalgo, J., y Tomás-Sábado, J. (2018). Relación entre inteligencia emocional, afecto negativo y riesgo suicida en jóvenes universitarios. Ansiedad y Estrés, 24(1), 18-23. https://doi.org/10.1016/j. anyes.2017.10.007

Jordan, P., y Lawrence, S. (2009). Emotional intelligence in teams: development and initial validation of the short version of the Workgroup Emotional Intelligence Profile (WEIP-S). Journal of Management \& Organization, 15(4), 452-469. https:// doi.org/10.5172/jmo.15.4.452

López-Zafra, E., Pulido, M., y Berrios, P. (2014). EQi-Versión corta (EQi-C). Adaptación y validación al español del EQ-i en universitarios. Boletín de Psicología, 110, 21-36.

Lyusin, D., y Ovsyannikova, V. (2016). Measuring two aspects of emotion recognition ability: accuracy vs. sensitivity. Learning and Individual Differences, 52, 129-136. doi: 10.1016/j.lindif.2015.04.010

Martí-Vilar, M., Serrano-Pastor, L., y González Sala, F. (2019). Emotional, cultural and cognitive variables of prosocial behaviour. Current Psychology, 38, 912-919 https://doi.org/10.1007/s12144-019-0168-9

Parker, J., Keefer, K., y Wood, L. (2011). Toward a brief multidimensional assessment of emotional intelligence: psychometric properties of the Emotional Quotient Inventory-Short Form. Psychological Assessment, 23(3), 762-777. https://doi. org/10.1037/a0023289 
Parker, J., Taylor, G., y Bagby, R. (2001). The relationship between emotional intelligence and alexithymia. Personality and Individual Differences, 30(1), 107-115. https://doi. org/10.1016/S0191-8869(00)00014-3

Pérez-Fuentes, M., Gázquez, J., Mercader, I., y Molero, M. (2014). Brief Emotional Intelligence Inventory for Senior Citizens (EQ-I-M20). Psicothema, 26(4), 524-530. https://doi.org/10.7334/psicothema2014.166

Petrides, K. V., Mikolajczak, M., Mavroveli, S., Sanchez-Ruiz, M. J., Furnham, A., y PérezGonzález, J. C. (2016). Developments in trait emotional intelligence research. Emotion Review, 8(4), 335-341. https://doi.org/10.1177/1754073916650493

Pincay-Aguilar, I., Candelario-Suarez, G., y Castro-Guevara, J. (2018). Inteligencia emocional en el desempeño docente. Psicología UNEMI, 2(2), 32-40. Recuperado de http://181.188.214.100/index.php/faso-unemi/article/view/699/595

Quiliano, M., y Quiliano, M. (2020). Inteligencia emocional y estrés académico en estudiantes de enfermería. Ciencia y Enfermería, 26(3), 1-9. https://doi.org/10.4067/ s0717-95532020000100203

Regner, E. (2008). Validez convergente y discriminante del Inventario de Cociente Emocional (EQ-i). Interdisciplinaria, 25(1), 29-51. Recuperado de https://www. redalyc.org/pdf/180/18025102.pdf

Rivera, E., Pons, J. I., Rosario-Hernández, E., y Ortiz, N. (2008). Traducción y adaptación para la población puertorriqueña del Inventario Bar-On de Cociente Emocional (Bar-On EQ-i): análisis de propiedades psicométricas. Revista Puertorriqueña de Psicología, 19, 148-182. Recuperado de https://dialnet.unirioja.es/servlet/ articulo?codigo $=4895882$

Ros, A., Filella, G., Ribes, R., y Pérez-Escoda, N. (2017). Análisis de la relación entre competencias emocionales, autoestima, clima de aula, rendimiento académico y nivel de bienestar en educación primaria. Revista Española de Orientación y Psicopedagogía, 28(1), 8-18. Recuperado de https://www.redalyc.org/ pdf/3382/338252055002.pdf

Ruvalcaba, N., Gallegos, J., y Fuerte, J. (2017). Competencias socioemocionales como predictoras de conductas prosociales y clima escolar positivo en adolescentes. Revista Interuniversitaria de Formación del Profesorado, 88(31.1), 77-90. Recuperado de https://www.redalyc.org/jatsRepo/274/27450136012/html/ index.html

Salovey, P., y Mayer, J. D. (1990). Emotional intelligence. Imagination, Cognition and Personality, 9, 185-211. https://doi.org/10.2190/DUGG-P24E-52WK-6CDG 
Samper, P., Mestre, V., y Malonda, E. (2014). Evaluación del rol de variables intelectuales y socioemocionales en la resolución de problemas en la adolescencia. Universitas Psychologica, 14(1), 15-26. https://doi.org/10.11144/Javeriana.upsy14-1.ervi

Serrano, C., y Andreu, Y. (2016). Inteligencia emocional percibida, bienestar subjetivo, estrés percibido, engagement y rendimiento académico en adolescentes. Revista de Psicodidáctica, 21(2), 357-374. https://doi.org/10.1387/RevPsicodidact.14887

Stanimirovic, R., y Hanrahan, S. (2012). Examining the dimensional structure and factorial validity of the Bar-On Emotional Quotient Inventory in a sample of male athletes. Psychology of Sport and Exercise, 13(1), 44-50, https://doi.org/10.1016/j.psychsport.2011.07.009

Ugarriza, N. (2001). La evaluación de la inteligencia emocional a través del Inventario de BarOn (I-CE) en una muestra de Lima Metropolitana. Persona, 4, 129-160. https:// www.redalyc.org/pdf/1471/147118178005.pdf

Weerdt, M., y Rossi, G. (2012). The Bar-On Emotional Quotient Inventory (EQ-i): Evaluation of psychometric aspects in the Dutch speaking part of Belgium. En G. Rossi (Ed.), Psychology Selected Papers (pp. 145-172). Londres: In Tech. doi: 10.5772/38875 